\title{
Corrigendum: Cell migration and antigen capture are antagonistic processes coupled by myosin II in dendritic cells
}

Mélanie Chabaud, Mélina L. Heuzé, Marine Bretou, Pablo Vargas, Paolo Maiuri, Paola Solanes, Mathieu Maurin, Emmanuel Terriac, Maël Le Berre, Danielle Lankar, Tristan Piolot, Robert S. Adelstein, Yingfan Zhang,

Michael Sixt, Jordan Jacobelli, Olivier Bénichou, Raphaël Voituriez, Matthieu Piel \& Ana-Maria Lennon-Duménil

Nature Communications 6:7526 doi: 10.1038/ncomms8526 (2015); Published 25 Jun 2015; Updated 14 Aug 2015

Previous work by Képiró et al. describing the photostable blebbistatin derivative para-nitroblebbistatin was inadvertently omitted from the reference list of this Article and should have been cited at instances where this inhibitor is referred to. For example, in the Results section, Képiró et al. should have been cited as follows: 'To test this hypothesis, we used a non-phototoxic form of Blebbistatin (para-nitroblebbistatin), which impairs cell migration (Supplementary Fig. 2c) and is compatible with GFP imaging (Képiró et al.)'.

Képiró, M. et al. para-Nitroblebbistatin, the non-cytotoxic and photostable myosin II inhibitor. Angew. Chem. Int. Ed. Engl. 53, $8211-8215$ (2014). 